\title{
Small Perturbation Solutions for Elliptic Equations
}

\section{OVIDIU SAVIN}

Department of Mathematics, University of California-Berkeley, Berkeley, California, USA

We consider viscosity solutions of fully nonlinear elliptic equations

$$
F\left(D^{2} u, D u, u, x\right)=0
$$

for which $u \equiv 0$ is a solution. We assume $F$ is smooth and uniformly elliptic only in a neighborhood of the points $(0,0,0, x)$, and show that $u$ is smooth in the interior if $\|u\|_{L^{\infty}}$ is sufficiently small.

Keywords Harnack inequality; Perturbation theory; Viscosity solutions.

Mathematics Subject Classification 35J60; 35B65.

\section{Introduction}

In this work we present a general regularity result for small perturbation solutions of elliptic equations. Our approach was motivated by the analysis of flat level sets in Ginzburg-Landau phase transitions models, which were considered in Savin (2003).

When dealing with uniformly elliptic equations of the form (1), the classical approach to regularity is to differentiate the equation with respect to a direction $e$. Then, $u_{e}$ solves the linearized equation which is treated as a linear equation with bounded measurable coefficients.

If $F$ is not uniformly elliptic in the whole domain, then, in order to bound the coefficients of the linearized equation, one needs a priori bounds on $u, D u$ and $D^{2} u$. This is the case in several problems such as the minimal surface or the MongeAmpere equation.

In this paper we discuss the regularity of "flat" viscosity solutions of (1). We prove interior $C^{2, \alpha}$ estimates for such solutions provided that $F$ is smooth and uniformly elliptic in a neighborhood of the set

$$
\left\{(0,0,0, x), x \in B_{1}\right\} .
$$

In particular, the solutions are classical in the interior.

Received August 1, 2005; Accepted September 1, 2005

Address correspondence to Ovidiu Savin, Department of Mathematics, University of California-Berkeley, Berkeley, CA 94720, USA; osavin@math.berkeley.edu 
Sometimes it is possible to prove interior estimates starting from the estimates for flat solutions. This is done using methods developed by Caffarelli (1987) and by Caffarelli and Wang (1993), in which local regularity results can be extended in the whole domain.

First we assume $F$ is measurable and prove Harnack inequality in the context of flat solutions (Theorem 1.1). Using this result we prove higher regularity once we know $F$ is smooth.

Let $\mathscr{S}$ be the space of $n \times n$ symmetric matrices. Assume that

$$
F: \mathscr{S} \times \mathbb{R}^{n} \times \mathbb{R} \times B_{1} \rightarrow \mathbb{R}
$$

is a function defined for pairs $(M, p, z, x)$, and satisfies the following hypothesis for $|p| \leq \delta,|z| \leq \delta$

H1) $F(\cdot, p, z, x)$ is elliptic, i.e.,

$$
F(M+N, p, z, x) \geq F(M, p, z, x) \text { if } N \geq 0,
$$

H2) $F(\cdot, p, z, x)$ is uniformly elliptic in a $\delta$ neighborhood of the origin with ellipticity constants $\Lambda \geq \lambda>0$, i.e.,

$$
\Lambda\|N\| \geq F(M+N, p, z, x)-F(M, p, z, x) \geq \lambda\|N\| \quad \text { if } N \geq 0,\|M\|,\|N\| \leq \delta .
$$

H3) constants are solutions of (1) i.e.,

$$
F(0,0, z, x)=0
$$

and $F$ is Lipschitz in $p$,

$$
|F(M, p, z, x)-F(M, q, z, x)| \leq \kappa|p-q|, \quad\|M\|,|p|,|q| \leq \delta .
$$

or

$\left.H 3^{\prime}\right)$ planes are solutions of (1) i.e.,

$$
F(0, p, z, x)=0
$$

Notice that we do not require any regularity for $F$ in the variables $z$ and $x$. Moreover, in hypothesis H3' we do not assume any regularity in the $p$ variable as well. Also we have no information about $F$ for $|p|,|z|>\delta$.

From $\mathrm{H} 2$ and $\mathrm{H} 3$ (or $\mathrm{H}^{\prime}$ ) we find that for $\|M\|,|p|,|q| \leq \delta$,

$$
\begin{aligned}
\Lambda\left\|M^{+}\right\|-\lambda\left\|M^{-}\right\|+\kappa|p| & \geq F(M, p, z, x) \\
& \geq \lambda\left\|M^{+}\right\|-\Lambda\left\|M^{-}\right\|-\kappa|p| \text { if }\|M\|, p, z \leq \delta .
\end{aligned}
$$

We recall the definition of viscosity solutions for second order elliptic equations.

Definition. The continuous function $u$ is a viscosity subsolution of (1) in $B_{1}$ if, whenever we can touch $u$ from above with a $C^{2}$ function $\varphi$ at some point $x \in B_{1}$, we have

$$
F\left(D^{2} \varphi(x), D \varphi(x), \varphi(x), x\right) \geq 0
$$


Similarly one can define the notion of viscosity supersolution.

We say that $u$ is a viscosity solution of (1) if it is both a viscosity subsolution and supersolution.

From now on we refer to positive constants depending on $n, \Lambda, \lambda, \kappa$ as universal constants.

Theorem 1.1 (Harnack Inequality). Suppose F satisfies H1, H2, H3 (or H3'). There exist universal constants $c_{0}$ small and $C_{0}$ large such that, if

$$
\begin{gathered}
u: B_{1} \rightarrow \mathbb{R}, \\
0 \leq u \leq c_{0} \delta \quad \text { in } B_{1}
\end{gathered}
$$

is a viscosity solution of (1), then

$$
u \leq C_{0} u(0) \text { in } B_{1 / 2}
$$

The proof of Theorem 1.1 follows the lines of the proof of Harnack inequality for linear equations. The fact that $F$ is nonlinear is compensated by the smallness condition (3) which heuristically linearizes $F$ near points $(0,0,0, x)$.

The classical Harnack inequality for linear equations follows from our theorem since in that case $\delta=\infty$.

As a consequence of Theorem 1.1 we obtain that the oscillation of a flat solution $u$, decreases in the interior.

Corollary 1.2. Let $u$ be a solution of (1) with

$$
u(0)=0, \quad\|u\|_{L^{\infty}\left(B_{1}\right)} \leq c_{0} \delta .
$$

Then

$$
\|u\|_{L^{\infty}\left(B_{1 / 2}\right)} \leq(1-v)\|u\|_{L^{\infty}\left(B_{1}\right)}
$$

where $v$ is small, universal.

This corollary allows us to use compactness methods for proving higher regularity of solutions.

Assume the function $F$ is more regular, and instead of $H 3$ we satisfy

H4) 0 is a solution of (1),

$$
F(0,0,0, x)=0
$$

and $F \in C^{2},\left\|D^{2} F\right\| \leq K$ in a $\delta$-neighborhood of the set

$$
\left\{(0,0,0, x), x \in B_{1}\right\} .
$$

We prove the following regularity theorem for small perturbation solutions under very general assumptions. 
Theorem 1.3. Suppose $F$ satisfies $\mathrm{H} 1, \mathrm{H} 2$ and $\mathrm{H} 4$.

There exist a constant $c_{1}$ (depending on $F$ ) such that if $u$ is a solution of (1) with

$$
\|u\|_{L^{\infty}\left(B_{1}\right)} \leq c_{1}
$$

then $u \in C^{2, \alpha}\left(B_{1 / 2}\right)$, and

$$
\|u\|_{C^{2, \alpha}\left(B_{1 / 2}\right)} \leq \delta
$$

In Sec. 2 we prove Theorem 1.1. In Sec. 3 we give some generalizations of Corollary 1.2 that we need later. In Sec. 4 we prove Theorem 1.3 and in Sec. 5 we discuss some applications of our results.

\section{Proof of Harnack Inequality}

The strategy of the proof is the following. We slide paraboloids of constant opening from below (above) till they touch for the first time the graph of $u$. These are the only points where we use that $u$ satisfies the equation.

We prove that at a contact point the values of $|u|,|\nabla u|,\left\|D^{2} u\right\|$ are small, and therefore $F$ becomes uniformly elliptic. From this we can estimate the Lebesgue measure of these touching points (see Lemma 2.1). Actually we show that, by increasing the opening of the sliding paraboloids, the set of touching points almost covers in measure $B_{1 / 2}$. This implies that $u \sim u(0)$ in $B_{1 / 2}$ except a set of very small measure.

If $u\left(x_{0}\right) \gg u(0)$ at some point $x_{0}$, we find by the same methods $u \gg u(0)$ in a set of positive measure, which contradicts the above estimate.

In Lemma 2.1 we obtain a measure estimate similar to the one obtained from the Alexandrov Bakelman Puci estimate for linear equations (see Caffarelli and Cabre, 1995).

Lemma 2.1. Let $u$ be a viscosity supersolution of (1) in $B_{1}$.

Let $a>0$ be small, $a \leq \delta / 2$. For each $y \in B \subset \bar{B}_{1}, B$ a closed set, we slide the paraboloid of opening $a$ and vertex $y$

$$
-\frac{a}{2}|x-y|^{2}+c_{y}
$$

from below till we touch the graph of $u$ for the first time. Suppose the set of all contact points, denoted by $A$, is included in a compact set of $B_{1} \cap\{u \leq \delta\}$. There exists a small universal constant $c$, such that

$$
|A| \geq c|B|
$$

By $|A|$ we denote the Lebesgue measure of the set $A$. Remark that $A$ is closed and therefore measurable.

Proof. First we consider the case when $u$ is uniformly semiconcave in $B_{1}$, i.e., the graph of $u$ admits at all the points a touching paraboloid of opening $b$ from above. 
For all $x \in A$ we can touch the graph at $(x, u(x))$ by a paraboloid of vertex $y$ and opening $a$ from below and a paraboloid of opening $b$ from above, hence $u$ is differentiable at $x$. The vertex $y$ is determined uniquely by

$$
y=x+\frac{1}{a} D u(x) .
$$

Moreover, it is easy to show that $\nabla u$ is Lipschitz on $A$ with

$$
[D u]_{\operatorname{Lip}(A)} \leq C(a, b) .
$$

Denote by $Z$ the set of points $z \in B_{1}$ for which $u$ can be approximated by a quadratic polynomial near $z$, i.e.,

$$
\begin{aligned}
u(x) & =P(z, x)+o\left(|x-z|^{2}\right) \\
& =u(z)+p(z) \cdot(x-z)+\frac{1}{2}(x-z)^{T} M(z)(x-z)+o\left(|x-z|^{2}\right) .
\end{aligned}
$$

Since $u$ is semiconcave, we know by Alexandrov's theorem (see Evans and Gariepy, 1992)

$$
\left|B_{1} \backslash Z\right|=0 .
$$

Next we want to show for $z \in A \cap Z$ that

$$
-a I \leq M(z) \leq C a I, \quad C \text { universal }
$$

The left inequality is obvious.

From (6) one can conclude that

$$
P(z, x)-\frac{\varepsilon}{2}|x-z|^{2}+\text { const }
$$

touches $u$ from below in a neighborhood of $z$ at some point $\tilde{x}$. Since $u$ is a viscosity supersolution of (1) we find

$$
F(M(z)-\varepsilon I, \tilde{p}, u(\tilde{x}), \tilde{x}) \leq 0
$$

for some $\tilde{p},|\tilde{p}|<2 a$. Assume by contradiction the right inequality in (7) doesn't hold. Then there exists a unit vector $e$ such that

$$
M(z) \geq C a e \otimes e-a I
$$

From H1 and (2)

$$
\begin{aligned}
0 & \geq F(M(z)-\varepsilon I, \tilde{p}, u(\tilde{x}), \tilde{x}) \\
& \geq F(C a e \otimes e-(a+\varepsilon) I, \tilde{p}, u(\tilde{x}), \tilde{x}) \\
& \geq \lambda(C a-a-\varepsilon)-\sqrt{n-1} \Lambda(a+\varepsilon)-\kappa|\tilde{p}|
\end{aligned}
$$

which is a contradiction if $C$ is sufficiently large. 
Now we can prove the conclusion of the lemma from (4)-(7),

$$
\begin{aligned}
|B| & =\int_{A}\left|\operatorname{det}\left(I+\frac{1}{a} D^{2} u(x)\right)\right| d x \\
& =\int_{A \cap Z} \operatorname{det}\left(I+\frac{1}{a} M(z)\right) d z \leq C|A \cap Z| .
\end{aligned}
$$

Now we treat the general case without assuming that $u$ is semiconcave.

We regularize $u$ by the standard method of inf-convolution, i.e.,

$$
u_{\varepsilon}(x)=\inf _{y \in B_{1}}\left\{u(y)+\frac{1}{\varepsilon}|y-x|^{2}\right\} .
$$

It is easy to check that $u_{\varepsilon}$ is semiconcave and $u_{\varepsilon} \rightarrow u$ uniformly on compact sets of $B_{1}$. Moreover, $u_{\varepsilon}$ is a viscosity supersolution for an appropriate elliptic equation.

Indeed, suppose $\varphi \in C^{2}$ touches $u_{\varepsilon}$ from below at $x_{0}$. Let $\tilde{x}_{0} \in B_{1}$ be such that

$$
\inf _{y \in B_{1}}\left\{u(y)+\frac{1}{\varepsilon}\left|y-x_{0}\right|^{2}\right\}=u\left(\tilde{x}_{0}\right)+\frac{1}{\varepsilon}\left|\tilde{x}_{0}-x_{0}\right|^{2} .
$$

Then

$$
\varphi\left(x+x_{0}-\tilde{x}_{0}\right)+u\left(\tilde{x}_{0}\right)-\varphi\left(x_{0}\right)
$$

touches $u$ from below at $\tilde{x}_{0}$, thus

$$
F\left(D^{2} \varphi\left(x_{0}\right), D \varphi\left(x_{0}\right), u\left(\tilde{x}_{0}\right), \tilde{x}_{0}\right) \leq 0 .
$$

By the first part of the proof we find

$$
\left|A_{\varepsilon}\right| \geq c|B|
$$

where $A_{\varepsilon}$ is the corresponding touching set for $u_{\varepsilon}$. It is easy to check

$$
\lim \sup A_{1 / k}=\bigcap_{m=1}^{\infty} \bigcup_{k=m}^{\infty} A_{1 / k} \subset A .
$$

In conclusion

$$
|A| \geq c|B|
$$

and the lemma is proved.

We denote by $A_{a}$ the set of points where we can touch $u$ from below with paraboloids of opening $a$ and vertex in $\bar{B}_{1}$ i.e.,

$$
\begin{aligned}
A_{a}:= & \left\{x \in B_{1} \mid u(x) \leq a \text { and } \exists y \in \bar{B}_{1}\right. \\
& \left.\quad \text { such that } \inf _{z \in B_{1}}\left(u(z)+\frac{a}{2}|z-y|^{2}\right)=u(x)+\frac{a}{2}|x-y|^{2}\right\}
\end{aligned}
$$

Notice that the sets $A_{a}$ are closed in $B_{1}$. 
Lemma 2.2. Let $B_{r}\left(x_{0}\right)$ be such that

$$
\begin{aligned}
& \bar{B}_{r}\left(x_{0}\right) \subset B_{1}, \\
& \bar{B}_{r}\left(x_{0}\right) \cap A_{a} \neq \emptyset .
\end{aligned}
$$

There exist positive universal constants $C, c$ such that if $a \leq C^{-1} \delta$, then

$$
\frac{\left|A_{C a} \cap B_{r / 8}\left(x_{0}\right)\right|}{\left|B_{r}\left(x_{0}\right)\right|} \geq c .
$$

Proof. Without loss of generality assume

$$
x_{1} \in B_{r}\left(x_{0}\right) \cap A_{a} \neq \emptyset .
$$

Otherwise we replace $r$ by $r+\varepsilon$ and the result follows by letting $\varepsilon \rightarrow 0$.

Denote by $y_{1} \in \bar{B}_{1}$ the vertex of the tangent paraboloid

$$
P\left(x, y_{1}\right):=u\left(x_{1}\right)+\frac{a}{2}\left|x_{1}-y_{1}\right|^{2}-\frac{a}{2}\left|x-y_{1}\right|^{2}
$$

that touches $u$ from below at $x_{1}$.

First we find a point $z \in \bar{B}_{r / 16}\left(x_{0}\right)$ such that

$$
u(z)-P\left(z, y_{1}\right) \leq \operatorname{Car}^{2}, \quad C \text { universal }
$$

Let $\varphi$ be the radially symmetric continuous function

$$
\varphi: \bar{B}_{1} \rightarrow \mathbb{R}, \quad \varphi(x)= \begin{cases}\alpha^{-1}\left(|x|^{-\alpha}-1\right), & \frac{1}{16} \leq|x| \leq 1 \\ \alpha^{-1}\left(16^{\alpha}-1\right), & |x| \leq \frac{1}{16}\end{cases}
$$

where $\alpha$ is a large universal constant.

Construct a function $\psi$ by adding a rescaling of the above function to the tangent paraboloid $P\left(x, y_{1}\right)$, i.e.,

$$
\psi: \bar{B}_{r}\left(x_{0}\right) \rightarrow \mathbb{R}, \quad \psi(x):=P\left(x, y_{1}\right)+\operatorname{ar}^{2} \varphi\left(\frac{x-x_{0}}{r}\right) .
$$

We claim that $\psi$ is a subsolution of (1) in the region

$$
r / 16<\left|x-x_{0}\right|<r
$$

Indeed, using (2) we find

$$
\begin{aligned}
F\left(D^{2} \psi, D \psi, \psi, x\right)= & F\left(a D^{2} \varphi-a I, a\left(y_{1}-x\right)+\operatorname{arD} \varphi, \psi, x\right) \\
\geq & \lambda a\left[(\alpha+1) t^{-\alpha-2}-1\right]-\sqrt{n-1} \Lambda a\left(1+t^{-\alpha-2}\right) \\
& -\kappa a\left(2+r t^{-\alpha-1}\right)>0,
\end{aligned}
$$


where

$$
t=\frac{\left|x-x_{0}\right|}{r}, \quad 1 / 16<t<1 .
$$

Now we slide the graph of $\psi$ from below till we touch the graph of $u$ for the first time. In other words we look for the point $z$ where

$$
\min _{x \in \bar{B}_{r}}(u-\psi)
$$

is realized.

If $x \in \partial \bar{B}_{r}\left(x_{0}\right)$ then

$$
u(x) \geq P\left(x, y_{1}\right)=\psi(x)
$$

Actually the value in (12) is negative since $x_{1} \in B_{r}\left(x_{0}\right)$ and

$$
u\left(x_{1}\right)-\psi\left(x_{1}\right)=P\left(x_{1}, y_{1}\right)-\psi\left(x_{1}\right)=-a r^{2} \varphi\left(\frac{x_{1}-x_{0}}{r}\right)<0 .
$$

Also, from the above considerations, we deduce that the minimum cannot be realized in the region given by (10). In conclusion,

$$
\begin{aligned}
z & \in \bar{B}_{r / 16}\left(x_{0}\right), \\
u(z) & <\psi(z) \leq P\left(z, y_{1}\right)+\text { Car }^{2}
\end{aligned}
$$

which proves (9).

Now we slide from below the family of paraboloids

$$
P\left(x, y_{1}\right)-C^{\prime} \frac{a}{2}|x-y|^{2}+c_{y}, \quad|y-z| \leq r / 64
$$

till they become tangent to the graph of $u$. The opening of the above paraboloid is $\left(C^{\prime}+1\right) a$ and the vertex

$$
\frac{C^{\prime}}{C^{\prime}+1} y+\frac{1}{C^{\prime}+1} y_{0}
$$

From (9) we find

$$
c_{y} \leq \operatorname{Car}^{2}+C^{\prime} \frac{a}{2}\left(\frac{r}{64}\right)^{2} .
$$

If $|x-z| \geq r / 16$ and $C^{\prime}$ is large, universal,

$$
\begin{aligned}
& P\left(x, y_{0}\right)-C^{\prime} \frac{a}{2}|x-y|^{2}+c_{y} \\
& \quad \leq P\left(x, y_{0}\right)-C^{\prime} \frac{a}{2}\left(\frac{r}{32}\right)^{2}+\operatorname{Car}^{2}+C^{\prime} \frac{a}{2}\left(\frac{r}{64}\right)^{2}<P\left(x, y_{0}\right) \leq u(x) .
\end{aligned}
$$


Thus, the contact points are inside $B_{r / 16}(z) \subset B_{r / 8}\left(x_{0}\right)$. From Lemma 2.1, (14) and (13) we obtain

$$
\left|A_{\left(C^{\prime}+1\right) a} \cap B_{r / 8}\left(x_{1}\right)\right| \geq c\left(\frac{C^{\prime}}{C^{\prime}+1}\right)^{n}\left|B_{r / 64}(z)\right|
$$

and the lemma is proved.

Next we prove a simple measure covering lemma.

Lemma 2.3. Suppose the closed sets $D_{k}$ have the following properties:

1)

$$
D_{0} \subset D_{1} \subset D_{2} \subset \cdots D_{k} \subset \bar{B}_{1 / 3}, \quad D_{0} \neq \emptyset
$$

2) for any $x, r$ such that

$$
\begin{aligned}
& B_{r}(x) \subset B_{1}, \quad B_{r / 8}(x) \subset B_{1 / 3}, \\
& \bar{B}_{r}(x) \cap D_{k} \neq \emptyset,
\end{aligned}
$$

we have

$$
\left|B_{r / 8}(x) \cap D_{k+1}\right| \geq c\left|B_{r}(x)\right| .
$$

Then

$$
\left|B_{1 / 3} \backslash D_{k}\right| \leq(1-\mu)^{k}\left|B_{1 / 3}\right|,
$$

where $\mu$ is a small positive constant depending on $c$.

Proof. Let $x_{0} \in B_{1 / 3}$ and

$$
r:=\operatorname{dist}\left(x_{0}, D_{k}\right)
$$

We claim that

$$
\left|B_{r / 3}\left(x_{0}\right) \cap D_{k+1}\right| \geq c_{1}\left|B_{1 / 3} \cap B_{r}\left(x_{0}\right)\right| .
$$

We denote

$$
x_{1}:=x_{0}-\frac{r}{6} \frac{x_{0}}{\left|x_{0}\right|}
$$

It is easy to check

$$
B_{r / 6}\left(x_{1}\right) \subset B_{r / 3}\left(x_{0}\right) \cap B_{1 / 3} .
$$

From property 2) above and

$$
\operatorname{dist}\left(x_{1}, D_{k}\right) \leq r+\frac{r}{6}=\frac{7}{6} r
$$


we conclude

$$
\left|B_{r / 6}\left(x_{1}\right) \cap D_{k+1}\right| \geq c r^{n} \geq c_{1}\left|B_{1 / 3} \cap B_{r}\left(x_{0}\right)\right|
$$

which proves (15).

For each $x \in B_{1 / 3} \backslash D_{k}$ we consider the ball of center $x$ and radiusv

$$
r=\operatorname{dist}\left(x, D_{k}\right) \text {. }
$$

From this family of balls we choose a Vitali cover i.e, balls $B_{r_{i}}\left(x_{i}\right)$ that cover $B_{1 / 3} \backslash D_{k}$ for which $B_{r_{i} / 3}\left(x_{i}\right)$ are disjoint (see Evans and Gariepy, 1992).

We have

$$
\begin{aligned}
\left|B_{1 / 3} \backslash D_{k}\right| & \leq \sum_{i}\left|B_{r_{i}}\left(x_{i}\right) \cap B_{1 / 3}\right| \\
& \leq c_{1}^{-1} \sum\left|B_{r_{i} / 3}\left(x_{i}\right) \cap\left(D_{k+1} \backslash D_{k}\right)\right| \leq c_{1}^{-1}\left|D_{k+1} \backslash D_{k}\right| .
\end{aligned}
$$

In conclusion,

$$
\left|B_{1 / 3} \backslash D_{k+1}\right| \leq\left|B_{1 / 3} \backslash D_{k}\right|-\left|D_{k+1} \backslash D_{k}\right| \leq\left(1-c_{1}\right)\left|B_{1 / 3} \backslash D_{k}\right|
$$

and the lemma is proved.

Proof of Theorem 1.1. We assume

$$
u(0)=\frac{a}{20}>0, \quad a<C^{-1} \delta
$$

and we want to prove that

$$
u(x) \leq C_{1} a \text { for } x \in B_{1 / 6} .
$$

Slide the polynomial of opening $a$ and vertex 0 from below till it touches the graph of $u$. Since $u \geq 0$, the contact points occur in $\bar{B}_{1 / 3}$, hence

$$
A_{a} \cap \bar{B}_{1 / 3} \neq \emptyset \text {. }
$$

Denote by

$$
D_{k}:=A_{a C^{k}} \cap \bar{B}_{1 / 3}
$$

where $C$ is the constant from Lemma 2.2. Moreover, Lemma 2.2 implies that the sets $D_{k}$ satisfy the hypothesis of Lemma 2.3 as long as $a C^{k+1} \leq \delta$, thus

$$
\begin{gathered}
\left|B_{1 / 3} \backslash D_{k}\right| \leq(1-\mu)^{k}\left|B_{1 / 3}\right| \quad \text { if } a C^{k+1} \leq \delta, \\
u(x) \leq a C^{k} \text { for } x \in D_{k} .
\end{gathered}
$$

Assume that there exists $x_{k} \in B_{1 / 6}$ such that

$$
u\left(x_{k}\right) \geq a C^{k+1}, \quad k \geq k_{0},
$$

where $k_{0}$ is a large universal constant. 
We want to show that there exists a point $x_{k+1}$ at distance

$$
b_{k}:=C_{0}(1-\mu)^{\frac{k}{n}}, \quad C_{0} \text { universal }
$$

from $x_{k}$ such that

$$
u\left(x_{k+1}\right) \geq a C^{k+2},
$$

provided that

$$
4 a C^{k+2} b_{k}^{-2}<C^{-1} \delta
$$

Assume by contradiction that $u$ is below $a C^{k+2}$ on $\partial B_{b_{k}}\left(x_{k}\right)$. In the cylinder $\left|x-x_{k}\right| \leq b_{k}$ we slide from above paraboloids

$$
\begin{gathered}
2 a C^{k+2} b_{k}^{-2}|x-y|^{2}+c_{y}, \\
\left|y-x_{k}\right| \leq C^{-1} b_{k}
\end{gathered}
$$

till they touch the graph of $u$.

The tangent paraboloid with vertex at $y$ is above

$$
u\left(x_{k}\right)+2 a C^{k+2} b_{k}^{-2}\left(|x-y|^{2}-\left|x_{k}-y\right|^{2}\right) \geq 2 a C^{k}+2 a C^{k+2} b_{k}^{-2}|x-y|^{2},
$$

hence, the contact points occur in the interior of $B_{b_{k}}\left(x_{k}\right)$ and are above $2 a C^{k}$. As long as (20) holds, we can apply Lemma 2.1 upside-down and obtain that the measure of the contact points is greater than

$$
c C_{0}^{n}(1-\mu)^{k}\left|B_{1}\right|>2(1-\mu)^{k}\left|B_{1 / 3}\right|
$$

If $k_{0}$ is chosen large such that

$$
\sum_{k=k_{0}}^{\infty} b_{k}<1 / 4-1 / 6
$$

then all the contact points lie inside $B_{1 / 4}$. This contradicts (16), (17), (22) and proves the existence of $x_{k+1}$.

Assume by contradiction that there exists a point $x_{k_{0}} \in B_{1 / 4}$ that satisfies (18) with $k=k_{0}$. Let $k_{*}$ be the largest value of $k$ for which (20) holds, hence

$$
\delta C^{-3}(1-\mu)^{\frac{2}{n}} \leq 4 a C^{k_{*}+2} b_{k_{*}}^{-2}
$$

By above, we find the corresponding points $x_{k} \in B_{1 / 4}$ for $k_{0} \leq k \leq k_{*}$. Instead of finding $x_{k_{*}+1} \in B_{b_{k_{*}}}\left(x_{k_{*}}\right)$ we try to find it in $B_{1 / 3}$. As in (21) we have that on $\partial B_{1 / 3}$ the tangent paraboloid is above

$$
2 a C^{k_{*}}+2 a C^{k_{*}+2} b_{k_{*}}^{-2}|x-y|^{2} \geq 2 a C^{k_{*}+2}\left(12 b_{k_{*}}\right)^{-2} \geq(12 C)^{-3}(1-\mu)^{\frac{2}{n}} \delta:=c_{0} \delta .
$$


From (3) we deduce that all contact points occur in the interior of $B_{1 / 3}$ which leads us as before to a contradiction. In conclusion

$$
u \leq a C^{k_{0}} \quad \text { in } B_{1 / 6}
$$

and the theorem is proved.

\section{Extensions of Corollary 1.2}

Before we start the proof of the main Theorem we need to prove Corollary 1.2 under slightly different assumptions on $u$ and $F$.

We assume $F \in C^{1}$, satisfies $\mathrm{H} 1, \mathrm{H} 2$ and

$\left.\mathrm{H} 3^{\prime \prime}\right) 0$ is a solution, i.e.,

$$
F(0,0,0, x)=0
$$

and

$$
\left|F_{p}(M, p, z, x)\right| \leq \kappa, \quad \text { for }\|M\|,|p|,|z| \leq \delta .
$$

Proposition 3.1. Suppose

$$
\|u\|_{L^{\infty}\left(B_{1}\right)} \leq \delta^{\prime} \leq c_{0} \delta, \quad u(0)=0 .
$$

There exists a small universal constant $v$ such that if

$$
-v \delta^{\prime} \leq F\left(D^{2} u, D u, u, x\right) \leq v \delta^{\prime} .
$$

and

$$
\left|F_{z}(M, p, z, x)\right| \leq v, \quad \text { for }\|M\|,|p|,|z| \leq \delta
$$

then

$$
\|u\|_{L^{\infty}\left(B_{1 / 2}\right)} \leq(1-v) \delta^{\prime}
$$

Proof. The important observation is that Lemmas 2.1 and 2.2 are still valid under the new hypothesis if $a \geq v \delta^{\prime}$. Indeed, the only things we have to check are (8) and (11). Instead of (8) we write

$$
\begin{aligned}
a & \geq v \delta^{\prime} \geq F(M(z)-\varepsilon I, \tilde{p}, u(\tilde{x}), \tilde{x}) \\
& \geq F(M(z)-\varepsilon I, \tilde{p}, 0, \tilde{x})-v \delta^{\prime} \\
& \geq \lambda(C a-a-\varepsilon)-\sqrt{n-1} \Lambda(a+\varepsilon)-2 \kappa a-a,
\end{aligned}
$$

and instead of (11)

$$
\begin{aligned}
F\left(D^{2} \varphi, D \varphi, \varphi, x\right) \geq & F\left(D^{2} \varphi, D \varphi, 0, x\right)-v \delta^{\prime} \\
\geq & \lambda a\left[(\alpha+1) t^{-\alpha-2}-1\right]-\sqrt{n-1} \Lambda a\left(1+t^{-\alpha-2}\right) \\
& -\kappa a\left(2+r t^{-\alpha-1}\right)-a>a \geq v \delta^{\prime} .
\end{aligned}
$$


Assume by contradiction that there exists a point $x_{0} \in B_{1 / 2}$ such that

$$
u\left(x_{0}\right) \geq-\delta^{\prime}+v \delta^{\prime}
$$

Using the same arguments as in the beginning of the proof of Theorem 1.1 (now the picture is translated $\delta^{\prime}$ down) we obtain

$$
\left|\left\{u \geq-\delta^{\prime}+C^{k} v \delta^{\prime}\right\} \cap B_{1 / 2}\right| \leq(1-\mu)^{k}\left|B_{1 / 2}\right|
$$

Now we slide from above the paraboloids

$$
16 \delta^{\prime}|x-y|^{2}+c_{y}, \quad|y| \leq 1 / 8
$$

Since $u(0)=0, u \leq \delta^{\prime}$, we find that the contact points belong to the set

$$
E:=\left\{u \geq-\delta^{\prime} / 4\right\} \cap B_{1 / 2}
$$

From Lemma 2.1 we have

$$
|E| \geq c_{1}, \quad c_{1} \text { universal. }
$$

We first choose $k$ such that

$$
(1-\mu)^{k}\left|B_{1 / 2}\right| \leq c_{1} / 2
$$

and then $v$ such that

$$
C^{k} v \leq 1 / 2
$$

Then (25), (26), and (27) contradict the fact that $(x, u(x))$ is a graph on $B_{1 / 2}$.

Corollary 3.2. [Rescaled version]

Suppose that in $B_{r}, r<1$,

$$
\|u\|_{L^{\infty}\left(B_{r}\right)} \leq \delta^{\prime} \leq c_{0} r^{2} \delta, \quad u(0)=0 .
$$

If

$$
\left|F\left(D^{2} u, D u, u, x\right)\right| \leq v \delta^{\prime} r^{-2}
$$

and

$$
\left|F_{z}(M, p, z, x)\right| \leq v r^{-2}, \quad \text { for }\|M\|,|p|,|z| \leq \delta
$$

then

$$
\|u\|_{L^{\infty}\left(B_{r / 2}\right)} \leq(1-v) \delta^{\prime}
$$


Proof. The function

$$
w(x):=r^{-2} u(r x), \quad x \in B_{1},
$$

satisfies

$$
\begin{gathered}
\|w\|_{L^{\infty}\left(B_{1}\right)} \leq \delta^{\prime} r^{-2} \leq c_{0} \delta, \\
\left|\widetilde{F}\left(D^{2} w, D w, w, x\right)\right| \leq v \delta^{\prime} r^{-2}
\end{gathered}
$$

where

$$
\widetilde{F}\left(D^{2} w, D w, w, x\right):=F\left(D^{2} w, r D w, r^{2} w, r x\right) .
$$

Since $\widetilde{F}$ satisfies H1, H2, H3" and

$$
\left|\widetilde{F}_{z}(M, p, z, x)\right|=r^{2}\left|F_{z}\left(M, r p, r^{2} z, r x\right)\right| \leq v,
$$

we can apply the above Proposition to $\widetilde{F}, w, \delta^{\prime} r^{-2}$ and obtain

$$
\|w\|_{L^{\infty}\left(B_{1 / 2}\right)} \leq(1-v) \delta^{\prime} r^{-2} .
$$

We iterate this result and obtain:

Corollary 3.3. Suppose F satisfies $\mathrm{H}^{\prime \prime}$, (23), (24). If for some $k$

$$
\|u\|_{L^{\infty}\left(B_{1}\right)} \leq \delta^{\prime} \leq c_{0} 2^{-2 k} \delta, \quad u(0)=0
$$

then

$$
\|u\|_{L^{\infty}\left(B_{\rho}\right)} \leq 2 \rho^{\beta} \delta^{\prime}, \quad \rho \geq 2^{-k-1}
$$

where $\beta$ is small, universal.

Proof. It suffices to prove by induction over $s$ that

$$
\|u\|_{L^{\infty}\left(B_{2}-s\right)} \leq(1-v)^{s} \delta^{\prime}, \quad s \leq k+1 .
$$

Assume the result is true for $s$. Then we can apply Corollary 3.2 with $r=2^{-s}$. Indeed,

$$
\begin{aligned}
\|u\|_{L^{\infty}\left(B_{2}-s\right)} & \leq(1-v)^{s} \delta^{\prime} \leq c_{0} 2^{-2 s} \delta, \\
\left|F\left(D^{2} u, D u, u, x\right)\right| & \leq v \delta^{\prime} \leq v(1-v)^{s} \delta^{\prime} 2^{2 s}, \\
\left|F_{z}(M, p, z, x)\right| & \leq v \leq v 2^{2 s},
\end{aligned}
$$

hence

$$
\|u\|_{L^{\infty}\left(B_{2^{-s-1}}\right)} \leq(1-v)^{s+1} \delta^{\prime}
$$




\section{Proof of Theorem $\mathbf{1 . 3}$}

Assume $F$ satisfies hypothesis $\mathrm{H} 1, \mathrm{H} 2$ and $\mathrm{H} 4$.

Definition. We denote by $P(N, q, t, x)$ the quadratic polynomial

$$
P(N, q, t, x):=\frac{1}{2} x^{T} N x+q \cdot x+t .
$$

Theorem 1.3 follows easily from the following proposition.

Proposition 4.1 (Quadratic Approximations). There exist small constants $\eta$, universal, and $r_{0}$ depending on $\delta, K$ and the universal constants such that, if

$$
\|u-P(N, q, t, x)\|_{L^{\infty}\left(B_{r}\right)} \leq r^{2+\alpha}, \quad r \leq r_{0},
$$

with

$$
F(N, q, t, 0)=0, \quad\|N\|,|q|,|t| \leq \delta / 2
$$

then,

$$
\begin{gathered}
\left\|u-P\left(N^{\prime}, q^{\prime}, t^{\prime}, x\right)\right\|_{L^{\infty}\left(B_{\eta r}\right)} \leq(\eta r)^{2+\alpha}, \\
F\left(N^{\prime}, q^{\prime}, t^{\prime}, 0\right)=0 .
\end{gathered}
$$

Proof. Define

$$
w: B_{1} \rightarrow[-1,1]
$$

such that

$$
u(x)=P(N, q, t, x)+r^{2+\alpha} w(x / r) .
$$

We want to show that

$$
\|w-P(\tilde{N}, \tilde{q}, \tilde{t}, x)\|_{L^{\infty}\left(B_{\eta}\right)} \leq \eta^{2+\alpha}
$$

and

$$
F\left(N+r^{\alpha} \widetilde{N}, q+r^{1+\alpha} \tilde{q}, t+r^{2+\alpha} \tilde{t}, 0\right)=0
$$

The function $w$ satisfies the following equation in the viscosity sense:

$$
F\left(N+r^{\alpha} D^{2} w, q+r N x+r^{1+\alpha} D w, P(N, q, t, r x)+r^{2+\alpha} w, r x\right)=0 .
$$

Define

$$
\begin{gathered}
\widetilde{F}(M, p, z, x):=r^{-\alpha}\left[F\left(N+r^{\alpha} M, q+r N x+r^{1+\alpha} p, P+r^{2+\alpha}(z+w(0)), r x\right)\right. \\
\left.-F\left(N, q+r N x, P+r^{2+\alpha} w(0), r x\right)\right] .
\end{gathered}
$$


The function $\widetilde{F}$ satisfies $\mathrm{H} 1, \mathrm{H} 2, \mathrm{H} 3^{\prime \prime}$ with

$$
\tilde{\delta}=r^{-\alpha} \delta
$$

Moreover, the function

$$
v:=w-w(0)
$$

satisfies

$$
\begin{aligned}
\left|\widetilde{F}\left(D^{2} v, D v, v, x\right)\right| & \leq C(K, \delta) r^{1-\alpha}, \\
\left|\widetilde{F}_{z}(M, p, z, x)\right| & \leq C(K, \delta) r^{2}, \quad\|M\|,|p|,|z| \leq \tilde{\delta} .
\end{aligned}
$$

Now we apply Corollary 3.3 to $\widetilde{F}, v$ with $\delta^{\prime}=2$. If

$$
\begin{gathered}
2 \leq c_{0} 2^{-2 k} \tilde{\delta}=c_{0} 2^{-2 k} r^{-\alpha} \delta, \\
C(K, \delta) r^{1-\alpha} \leq 2 v,
\end{gathered}
$$

then

$$
\|v\|_{L^{\infty}\left(B_{\rho}\right)} \leq 4 \rho^{\beta} \text { for } \rho \geq 2^{-k-1} .
$$

We obtain similar inequalities if we consider functions

$$
w-w\left(x_{0}\right), \quad x_{0} \in B_{1 / 2}
$$

In conclusion, if $x_{1}, x_{2} \in B_{1 / 2}$ we have

$$
\left|w\left(x_{1}\right)-w\left(x_{2}\right)\right| \leq 4\left|x_{1}-x_{2}\right|^{\beta}, \quad \text { for }\left|x_{1}-x_{2}\right| \geq C_{0} \delta^{-1 / 2} r^{\alpha / 2}
$$

hence, $w$ has a Holder modulus of continuity outside a $C_{0} \delta^{-1 / 2} r^{\alpha / 2}$ neighborhood of the origin.

Now we can use the compactness argument.

Assume by contradiction the Proposition is false. Then, there exists a sequence of $r_{k} \rightarrow 0$ and corresponding $F_{k}, w_{k}, N_{k}, q_{k}, t_{k}$ for which the conclusion doesn't hold. From (28) we can extract a convergent subsequence that converges to a Holder continuous function in $B_{1 / 2}$. Without loss of generality we assume $\left\|D^{2} F_{k}\right\| \leq K$,

$$
\begin{aligned}
& F_{k} \rightarrow F_{*}, \quad D F_{k} \rightarrow D F_{*} \quad \text { uniformly, } \\
& w_{k} \rightarrow w_{*} \quad \text { uniformly in } B_{1 / 2}, \\
& N_{k} \rightarrow N_{*}, \quad q_{k} \rightarrow q_{*}, \quad t_{k} \rightarrow t_{*} .
\end{aligned}
$$

We claim that $w_{*}$ is in $B_{1 / 2}$ a viscosity solution of the constant coefficients linear equation

$$
D_{M} F_{*}\left(N_{*}, q_{*}, t_{*}, 0\right): D^{2} w_{*}=0 .
$$


Assume by contradiction that we can touch $w_{*}$ from below at $x_{*}$ by a smooth function $\varphi+\varepsilon\left|x-x_{*}\right|^{2}$ and

$$
D_{M} F_{*}\left(N_{*}, q_{*}, t_{*}, 0\right): D^{2} \varphi\left(x_{*}\right)>\varepsilon>0 .
$$

Then

$$
\varphi+\text { const. }
$$

touches $w_{k}$ from below at $x_{k}, x_{k} \rightarrow x_{*}$. We have

$$
\begin{aligned}
0 \geq & {\left[F_{k}\left(N_{k}+r_{k}^{\alpha} D^{2} \varphi, q_{k}+r_{k} N_{k} x_{k}+r_{k}^{1+\alpha} D \varphi, P_{k}+r_{k}^{2+\alpha}(\varphi+c), r_{k} x_{k}\right)\right.} \\
& \left.\quad-F_{k}\left(N_{k}, q_{k}, t_{k}, 0\right)\right] r_{k}^{-\alpha} \\
\geq & D_{M} F_{k}\left(N_{k}, q_{k}, t_{k}, 0\right): D^{2} \varphi\left(x_{k}\right) \\
& \quad-C(\varphi, K) \max \left\{r_{k}^{1-\alpha}, r_{k}^{\alpha}\right\}>\varepsilon / 2 \text { as } k \rightarrow \infty
\end{aligned}
$$

contradiction, and the claim is proved.

From (29) we conclude the existence of $N^{\prime}, q^{\prime}, t^{\prime}$ such that

$$
\begin{gathered}
D_{M} F_{*}\left(N_{*}, q_{*}, t_{*}, 0\right): N^{\prime}=0, \\
\left\|w_{*}-P\left(N^{\prime}, q^{\prime}, t^{\prime}, x\right)\right\|_{L^{\infty}\left(B_{\eta}\right)} \leq \eta^{2+\alpha} / 3,
\end{gathered}
$$

for some $\eta$ depending on $n, \lambda, \Lambda$.

Now we solve for $\widetilde{N}_{k}$,

$$
\widetilde{N}_{k}=N^{\prime}+s_{k} I,
$$

the equation

$$
F_{k}\left(N_{k}+r_{k}^{\alpha} \tilde{N}_{k}, q_{k}+r_{k}^{1+\alpha} q^{\prime}, t_{k}+r_{k}^{2+\alpha} t^{\prime}, 0\right)=0 .
$$

It suffices to show that for large $k$

$$
s_{k} \leq \eta^{\alpha} / 3
$$

since then, $w_{k}$ satisfies the conclusion

$$
\left\|w_{k}-P\left(\widetilde{N}_{k}, q^{\prime}, t^{\prime}, x\right)\right\|_{L^{\infty}\left(B_{\eta}\right)} \leq \eta^{2+\alpha}
$$

We have

$$
\begin{aligned}
& r_{k}^{-\alpha} F_{k}\left(N_{k}+r_{k}^{\alpha} N^{\prime}, q_{k}+r_{k}^{1+\alpha} q^{\prime}, t_{k}+r_{k}^{2+\alpha} t^{\prime}, 0\right) \\
& \quad=D_{M} F_{k}\left(N_{k}, q_{k}, t_{k}, 0\right): N^{\prime}+O\left(r_{k}^{\alpha}\right) \rightarrow 0 \text { as } k \rightarrow \infty
\end{aligned}
$$

This implies $s_{k} \rightarrow 0$ and the proposition is proved. 


\section{Further Remarks}

\subsection{Curvature Equations}

The methods used to prove Theorem 1.1 can be modified appropriately for certain type of equations. We illustrate this by considering geometric equations depending on the curvatures of the graph of $u$ instead of $D^{2} u$.

Denote by $I I u(x)$ the second fundamental form of the graph of $u$ at $x$. In order to represent $\operatorname{IIu}(x)$, we choose an orthonormal basis $e_{1}, e_{2}, \ldots, e_{n}$ at $x$ such that $\nabla u(x)$ points in the $e_{n}$ direction. The normal to the graph is

$$
v=\frac{e_{n+1}}{\sqrt{1+|D u|^{2}}}-\frac{|D u| e_{n}}{\sqrt{1+|D u|^{2}}}:=\alpha e_{n+1}-\beta e_{n} .
$$

On the tangent plane generated by $e_{1}, e_{2}, \ldots, e_{n-1}, f_{n}$ where

$$
f_{n}:=\beta e_{n+1}+\alpha e_{n},
$$

the symmetric matrix $I I u(x)$ is given by

$$
[\operatorname{IIu}(x)]_{i j}= \begin{cases}\alpha u_{i j}(x), & 1 \leq i, j \leq n-1 \\ \alpha^{2} u_{i n}(x), & 1 \leq i \leq n-1, j=n \\ \alpha^{3} u_{n n}(x), & i=j=n\end{cases}
$$

As in the case of the Hessian, we consider the class of continuous functions $S(\lambda, \Lambda)$ for which the positive curvatures and the negative curvatures are balanced, i.e

$$
\frac{\lambda}{\Lambda}\left\|I I u^{-}\right\| \leq\left\|I I u^{+}\right\| \leq \frac{\Lambda}{\lambda}\left\|I I u^{-}\right\|
$$

in the viscosity sense. More precisely, if we touch $u$ from below at $x$ by a smooth function $\varphi$, then

$$
\left\|I I \varphi(x)^{+}\right\| \leq \frac{\Lambda}{\lambda}\left\|I I \varphi(x)^{-}\right\|,
$$

and if we touch from above we satisfy the left inequality in (30).

Obviously, Theorem 1.1 is valid for functions $u \in S(\lambda, \Lambda)$ for some $\delta_{0}$ small, universal.

Actually, in this case it is more natural to slide balls in the $e_{n+1}$ direction instead of paraboloids.

Lemma 5.1. Suppose $u \in S(\lambda, \Lambda)$, and let $a>0$ be arbitrary. For each $y \in B, B a$ closed set, we slide the ball of opening $1 /$ a and vertex $y+t_{n+1}$ from below till we touch the graph of $u$ for the first time. Suppose the set of all contact points, denoted by A, is included in a compact set of $B_{1}$. Then,

$$
|A| \geq c|B|, \quad c \text { universal. }
$$


The proof is similar to the proof of Lemma 2.1 and we omit the details. Instead of (4) we write

$$
y=x+\frac{1}{a} \frac{D u(x)}{\sqrt{1+|D u|^{2}}}
$$

and then we replace $D^{2} u$ (and $M(z)$ ) by IIu.

One advantage of sliding balls is that for small radius the contact points occur in the interior of $B_{1}$. Using this observation, we can replace the assumption that $u$ is bounded above in Theorem 1.1 by the assumption that $u(0)$ is small.

Theorem 5.2. Let $u \in S(\lambda, \Lambda)$. If $u \geq 0$ in $B_{1}$, and

$$
u(0) \leq \delta \quad \delta \text { universal, }
$$

then

$$
u \leq C_{0} u(0) \text { in } B_{1 / 2} \text {. }
$$

We give the idea of the proof.

It suffices to show that $u$ is bounded by a small constant $\delta_{0}$ in the interior, since then we can apply Theorem 1.1. If for some $x_{0} \in B_{1 / 4}, u\left(x_{0}\right)>\delta_{0}$, then, by sliding balls of radius $1 / 4$ and centers that project near $x_{0}$, we obtain

$$
\left|\left\{u>\delta_{0} / 2\right\} \cap B_{1 / 2}\right| \geq c_{0}\left(\delta_{0}\right) .
$$

Since $u \geq 0$ in $B_{1}$ and $u(0) \leq \delta$, we have from the proof of Theorem 1.1

$$
\left|\left\{u>C^{k} \delta\right\} \cap B_{1 / 2}\right| \leq(1-\mu)^{k}\left|B_{1 / 2}\right| .
$$

This contradicts (31) if we first choose $k$ large, and then $\delta$ small enough.

\subsection{Minimal Boundaries}

Let $\Omega \subset \mathbb{R}^{n+1}$ be an open set and $E$ be a measurable set. The perimeter of $E$ in $\Omega$ is defined as

$$
P(E, \Omega)=\sup \left|\int_{E} \operatorname{div} g d x\right|,
$$

where the supremum is taken over all vector fields $g \in C_{0}^{1}(\Omega)$ with $\|g\| \leq 1$.

We say that $E$ is a set with minimal perimeter in $\Omega$ if

$$
P(E, \Omega) \leq P(F, \Omega)
$$

whenever $E$ and $F$ coincide outside a compact set included in $\Omega$.

If $E$ has $C^{1}$ boundary, then the perimeter is the $n$ dimensional Hausdorff measure of $\partial E$,

$$
P(E, \Omega)=\mathscr{H}^{n}(\partial E \cap \Omega)
$$


An important result in the regularity theory for sets with minimal perimeter is the Flatness Theorem of De Giorgi (see Giusti, 1984):

Theorem (De Giorgi). Suppose the open set $E$ with

$$
\left\{x_{n+1} \leq-\delta\right\} \cap \mathscr{C} \subset E \cap \mathscr{C} \subset\left\{x_{n+1} \leq \delta\right\}
$$

has minimal perimeter in the cylinder

$$
\mathscr{b}:=\{|x|<1\} \times\left\{\left|x_{n+1}\right|<1\right\} .
$$

Then $\partial E \cap\{|x| \leq 1 / 2\}$ is a $C^{\infty}$ surface, if $\delta$ is small, universal.

Even though $\partial E$ is not a graph in the $e_{n+1}$ direction, it satisfies the mean curvature equation

$$
\operatorname{div} \frac{D u}{\sqrt{1+\mid D u \|^{2}}}=\operatorname{tr}[I I u]=0
$$

in the viscosity sense (see Caffarelli and Cordoba, 1993). That is, if we touch $\partial E$ with the graph of a smooth function from the interior (exterior) of $E$, then we have the corresponding inequality.

With the methods developed, we give a different proof of De Giorgi's theorem. The main step is the following version of Harnack inequality for $\partial E$.

Lemma 5.3. Suppose $E$ satisfies the above hypothesis and $0 \in \partial E$. Then

$$
\partial E \cap\{|x| \leq 1 / 2\} \subset\left\{\left|x_{n+1}\right| \leq(1-v) \delta\right\} .
$$

We give an outline of the proof.

Let

$$
F:=\left\{|x| \leq 1 / 2, x_{n+1} \geq-\delta\right\} .
$$

From the minimality of $P(E, \mathscr{C})$ we have

$$
P(E, \mathscr{C}) \leq P(E \backslash F, \mathscr{C})
$$

hence,

$$
P(E,\{|x|<1 / 2\}) \leq \mathscr{H}^{n}(\bar{E} \cap \partial F) \leq C(n) \delta+\left|B_{1 / 2}\right| .
$$

Assume by contradiction that

$$
\partial E \cap\{|x| \leq 1 / 2\} \cap\left\{x_{n+1} \leq-(1-v) \delta\right\} \neq \emptyset .
$$

As in the proof of Theorem 1.1 we obtain

$$
\left|D_{k} \cap B_{1 / 2}\right| \geq\left(1-(1-\mu)^{k}\right)\left|B_{1 / 2}\right|
$$


where $D_{k}$ is the projection along $e_{n+1}$ of the contact set of $\partial E$ with paraboloids of opening $C^{k} v \delta$ that stay below

$$
x_{n+1} \leq-\delta+C^{k} v \delta
$$

Then, we conclude that

$$
P\left(E,\{|x|<1 / 2\} \cap\left\{x_{n+1}<-\delta+2 C^{k} v \delta\right\}\right) \geq\left(1-(1-\mu)^{k}\right)\left|B_{1 / 2}\right| .
$$

Since $0 \in \partial E$, we obtain by sliding parabolids from above that

$$
P\left(E,\{|x|<1 / 2\} \cap\left\{x_{n+1}>-\delta / 2\right\}\right) \geq c_{0}, \quad c_{0} \text { universal. }
$$

If $C^{k} v<1 / 4$, the last two inequalities imply that

$$
P(E,\{|x|<1 / 2\}) \geq c_{0}+\left(1-(1-\mu)^{k}\right)\left|B_{1 / 2}\right|
$$

which contradicts (32) for small $\delta$ and large $k$.

Now we can use compactness arguments and prove an "improvement of flatness" lemma for $\partial E$.

Lemma 5.4. Assume $E$ satisfies the above hypothesis, $0 \in \partial E$. There exists $\eta$ universal, such that in some new system of coordinates $\left(\tilde{x}, \tilde{x}_{n+1}\right)$ we have

$$
\partial E \cap\{|\tilde{x}| \leq \eta\} \subset\left\{\left|\tilde{x}_{n+1}\right| \leq \eta \delta / 2\right\} .
$$

This lemma implies $\partial E$ is $C^{1, \alpha}$ in the interior, and therefore analytic by the classical regularity theory for elliptic equations.

\subsection{Neumann Boundary Conditions}

We mention that our methods can also be applied for Neumann boundary conditions. Let $\Omega \subset B_{1}$ be a Lipschitz domain with $\partial \Omega$ given by a Lipschitz graph in the $e_{n}$ direction, i.e there exists $b>0$ such that for all $y \in \partial \Omega \cap B_{1}$,

$$
\left\{x\left|(x-y) \cdot e_{n} \geq b\right| x-y \mid\right\} \cap B_{1} \subset \Omega .
$$

Assume the continuous function $u$,

$$
u: \bar{\Omega} \cap B_{1} \rightarrow \mathbb{R},
$$

is a viscosity solution of

$$
\begin{aligned}
F\left(D^{2} u, D u, u, x\right)=0 & \text { in } \Omega, \\
u_{\tau(x)}(x)=0 & \text { on } \partial \Omega,
\end{aligned}
$$

where $\tau(x)$ is a unit vector satisfying

$$
e_{n} \cdot \tau(x) \geq \beta>b .
$$


That is, if $\varphi$ is smooth and touches $u$ from above at $x \in \Omega$ then

$$
\begin{array}{r}
F\left(D^{2} u, D \varphi, \varphi, x\right) \geq 0 \text { if } x \in \Omega, \\
\varphi_{\tau(x)}(x) \geq 0 \quad \text { if } x \in \partial \Omega .
\end{array}
$$

Similarly, if $\varphi$ touches $u$ from below then it satisfies the reversed inequalities.

If $0 \in \bar{\Omega}$, then one can prove that Theorem 1.1 is still valid with $c_{0}$ depending also on $b$ and $\beta$. Moreover, if $\partial \Omega \in C^{2, \alpha}$ and $\tau \in C^{1, \alpha}$, then Theorem 1.3 is also satisfied.

\section{References}

Caffarelli, L. A. (1987). A Harnack inequality approach to the regularity of free boundaries. I. Lipschitz free boundaries are C1, $\alpha$. Rev. Mat. Iberoamericana 3(2):139-162.

Caffarelli, L., Cordoba, A. (1993). An elementary regularity theory of minimal surfaces. Differential Integral Equations 6(1):1-13.

Caffarelli, L. A., Wang, L. (1993). A Harnack inequality approach to the interior regularity of elliptic equations. Indiana Univ. Math. J. 42(1):145-157.

Caffarelli, L., Cabre, X. (1995). Fully Nonlinear Elliptic Equations. Colloquium Publications 43. Providence, RI: American Mathematical Society.

Evans, L. C., Gariepy, R. F. (1992). Measure Theory and Fine Properties of Functions. Studies in Advanced Mathematics. Boca Raton, FL: CRC Press.

Giusti, E. (1984). Minimal Surfaces and Functions of Bounded Variation. Basel-Boston: Birkhauser Verlag.

Savin O. (2003). Phase Transitions: Regularity of Flat Level Sets. PhD Thesis, UT Austin (2003). 